\title{
Hallazgos iniciales de la evaluación de Holguín para su desarrollo como destino turístico inteligente
}

Initial findings from the evaluation of Holguin for its development as a smart tourist destination

MSc. Justa R. Medina Labrada. ${ }^{1}$, Lic. Elizabeth del Carmen Pérez Ricardo. ${ }^{2}$ \& MSc. Ernesto Batista Sánchez. ${ }^{3}$

\begin{abstract}
Presently, the development of smart tourist destinations represents a priority, which constitutes, at the same time, an essential tool for the sector progress as wells as for society in general. For a tourist destination to be considered as a smartly managed destination, numerous and constantly evolving factors should be taken into account: improvement of internal management processes as well as marketing processes; supplying network management; constant research which allows adaptation not only to demand but also to clients' cultural characteristics. These elements can be grouped into five main fields: technology, innovation, governance, sustainability and accessibility. The effect of these aspects on the continuous improvement of destinations and their capacity to compete in a global economy depends on good management. Holguin is an important tourist destination on the northeastern part of the Island, which possesses valuable assets such as accessibility, relevant cultural and natural tourist attractions plus the required infrastructure for its tourist development. Therefore, this research aims at assessing tourist market management in Holguín destination in view of its development as smart tourist destination. The determination of variables to be used in the assessment was carried out through an analysis of similarities. Subsequently, a case study was applied in order to specify the aspects showing favorable outcomes as well as the indicators demanding changes so that Holguín may develop as a smart tourism destination.
\end{abstract}

Key Words: Smart Tourist Destination, Holguin, Sustainable Development.

\section{Resumen}

El desarrollo de destinos turísticos inteligentes es un imperativo en el contexto actual y constituye una herramienta fundamental en el progreso del sector y de la sociedad, en

\footnotetext{
1 jmedina@uho.edu.cu

2 elizabeth.ricardo@uho.edu.cu
} 
general. Para considerar un destino turístico con una gestión inteligente deben tenerse en cuenta un grupo de factores, en continua evolución, entre los que se incluyen: una mejora en sus procesos tanto de gestión interna, como de comercialización; gestión con proveedores; una investigación continua para adaptar los productos y servicios no sólo a la demanda de sus clientes, sino también a sus gustos y costumbres, entre otros. Estos factores pueden ser agrupados en cinco ámbitos fundamentales: Tecnología, innovación, gobernanza, sostenibilidad y accesibilidad, los que bien gestionados, contribuyen a la continua mejora del destino para poder competir en una economía cada vez más globalizada. El destino Holguín posee gran importancia para el turismo en el Nororiente de Cuba, al disponer de buena accesibilidad; significativos atractivos culturales, históricos y naturales y la infraestructura necesaria para su desarrollo turístico; por lo que la presente investigación se planteó como objetivo evaluar la gestión de Holguín para su desarrollo como destino turístico inteligente, para lo cual se determinaron las variables a emplear a través de un análisis de similitudes y luego se empleó el caso de estudio para precisar los aspectos con resultados favorables y los indicadores a transformar para que Holguín se desarrolle como destino inteligente.

Palabras Clave: Destino Turístico Inteligente, Holguín, Desarrollo Sostenible.

\section{Introducción}

El turismo se encuentra en un momento de cambio acelerado por la emergencia e impacto directo de multitud de nuevas realidades que han cambiado por completo las reglas del juego. Muchos de dichos cambios tienen su origen directo en la implantación de tecnologías que transforman los roles tradicionalmente asignados a los distintos agentes implicados en el turismo. Así, las tecnologías de la información y la comunicación (TIC) se han convertido en un motor de cambio constante, alterando el modo en que entendemos los destinos, el funcionamiento de las empresas o el comportamiento de los turistas. En este contexto de cambio acelerado, durante el último lustro el concepto de destino turístico inteligente, en lo adelante DTI, bajo el paraguas del acuñado como 'turismo inteligente' o smart tourism, ha calado con fuerza en el discurso institucional, académico y empresarial, derivado principalmente de las tesis de la ciudad inteligente (FemeniaSerra \& Ivars-Baidal, 2018).

Hay coincidencia en esta visión por parte de varios estudiosos de la gestión de los destinos. (Ivars-Baidal, Solsona-Monzonís, \& Giner-Sánchez, 2016) plantean que "E1 interés por los destinos inteligentes coincide con un contexto de cambio estructural en el turismo (impacto de las tecnologías de la información y la comunicación, nuevos patrones de comportamiento de la demanda, aparición de nuevos modelos de negocio, mayor sensibilidad ambiental, etc.), que requiere nuevos enfoques de gestión, de acuerdo con una serie de objetivos:

- Garantizar la calidad ambiental y la sostenibilidad de los destinos

- Hallar nuevas vías de competitividad en el escenario turístico actual

- Enriquecer y diferenciar las experiencias turísticas que ofrecen los destinos

- Reforzar y comunicar los atractivos del territorio 
- Aprovechar la alta penetración de las tecnologías de la información y la comunicación (TIC) en la producción y el consumo turísticos.

También (Fernández Alcantud, 2017) plantea que "más allá de considerar la aportación sin duda ineludible de las empresas de tecnología, hay que considerar las aportaciones desde otros ámbitos empresariales, pero también desde la contribución de otros agentes (comunidad local, turistas, administraciones públicas, universidades y/o centros de investigación). Todos estos agentes pueden verse a su vez beneficiados por su participación en el ecosistema que supone el DTI".

Este autor remarca además que "el DTI supone fijar una estrategia de revalorización del destino mediante un mejor aprovechamiento de los atractivos naturales y culturales, la creación de recursos innovadores, y la mejora en la eficiencia en los procesos de producción y distribución. Un destino capaz de innovar implementando nuevos sistemas que faciliten el análisis de múltiples fuentes de información (big data, business intelligence) y les permita ser más eficientes y competitivos, en el que las empresas y gestores del destino evolucionen para dar respuesta al nuevo viajero en todo el ciclo del viaje".

El desarrollo de destinos turísticos inteligentes está directamente relacionado con las tendencias actuales de la demanda que incluyen aspectos como: gran protagonismo de las tecnologías de la información y la comunicación para interactuar con las marcas; viajes activos, con múltiples dispositivos, canales y experiencias; personalización de los servicios; participación de los clientes en la cocreación de experiencias y gran sensibilidad a la protección del medio ambiente y a los beneficios sociales que reporta cada viaje . La propia tecnología está creando un viajero más exigente, hiperinformado, permanentemente conectado, que busca un servicio personalizado y experiencias auténticas y en definitiva una oferta turística diferencial coherente con el entorno natural, social y cultural.

Desde el punto de vista de (Salessi, 2017)“Una ciudad innovadora, y un destino turístico que se precie de autoproclamarse inteligente debería promover espacios que acerquen inclusivamente a turistas con residentes, en el marco de una propuesta turística consecuente con una serie de prestaciones de productos y servicios turísticos creativos con los cuales legitimar ese encuentro: transporte y conectividad, sistemas de alojamiento, información e interpretación turística, servicios, aplicaciones de destinos, restauración, recreación, entre otros".

Por todo ello "los beneficios y ventajas competitivas que supone para cualquier territorio la adopción del modelo de destino turístico inteligente, son notables ya que no sólo revierte en el ámbito turístico, sino en muchos otros sectores y siendo conscientes de que la situación de partida de cada municipio es muy distinta, resulta difícil proponer un esquema de trabajo general. No obstante, en todos los casos es necesario realizar ese primer diagnóstico y, a partir de su resultado, trazar un plan de acción, que aborde las áreas de mejora, alineado con el posicionamiento o estrategia que quiera desarrollar el destino en el futuro" (López et al., 2015). 
El destino Holguín es uno de los destinos cubanos más frecuentados por el turismo nacional y extranjero que visita el Oriente de Cuba. Posee importantes atractivos para el desarrollo del turismo, entre los que se destacan sus playas de arenas blancas y finas como Guardalavaca, Pesquero y Esmeralda; espacios naturales reconocidos como el Parque Natural Alejandro de Humboldt en el cual se encuentra la Reserva de la biosfera Cuchillas del Toa, área protegida de alto valor florístico y faunístico, que por su endemismo y grado de conservación fue declarada por la UNESCO como Patrimonio de la Humanidad.

También está Cayo Bariay, lugar del desembarco de Cristóbal Colón en 1492, situado en el municipio Freyre y Birán, sitio natal del Comandante Fidel Castro Ruz. Además, cuenta con recursos patrimoniales en la ciudad de Gibara, ciudad marinera que conserva en la actualidad el más notable conjunto arquitectónico colonial de la provincia de Holguín y con peculiar cultura culinaria. Así mismo, en el municipio Banes, se puede acceder al Chorro de Maíta, uno de los más relevantes recintos funerarios y ceremoniales precolombinos descubierto en las Antillas y el Museo Indocubano Baní, con la más completa colección de piezas de cerámica y objetos ornamentales y de trabajo de los primeros habitantes de las Antillas.

Por otra parte, en la ciudad de Holguín se desarrollan eventos culturales propios y de gran convocatoria nacional e internacional como Las Romerías de Mayo, que, a través del arte joven, recrea las mejores tradiciones y que acoge al Festival Mundial de Juventudes cada año; así como la Fiesta de la Cultura Iberoamericana. Además, en la ciudad está la Loma de la Cruz, elevación con acceso vehicular o a través de sus 456 escalones, desde donde el Papa Francisco extendió su bendición a todos los holguineros durante su visita en 2015.

También existe una infraestructura conformada por hoteles de tres, cuatro y cinco estrellas pertenecientes a las compañías ISLAZUL, CUBANACAN y GAVIOTA, numerosas casas de renta, paladares, centros nocturnos, tiendas, servicios de transporte diversos; así como una imagen propia que es reconocida por los clientes de varios continentes.

Dado que Holguín es un destino importante del Oriente de Cuba, a partir del número de clientes que la visitan anualmente; así como del conjunto de atractivos que posee, la infraestructura turística disponible y que en él se encuentra el acceso aéreo a los diferentes polos turísticos de la región y, por otra parte, los destinos turísticos inteligentes son clave para el desarrollo sostenible y contribuyen, no solo a introducir mejoras para el sector turístico, sino también para el conjunto de la sociedad, la presente investigación se propuso el objetivo de evaluar a Holguín para su desarrollo como destino turístico inteligente.

\section{Revisión de la literatura}

En los últimos años se han materializado, tanto en la investigación como en la práctica, múltiples modelos de la gestión de destinos turístico. El crecimiento del interés por este tema refleja una preocupación no solamente por el desarrollo del turismo sino también por la necesidad de gestionarlo con el fin de asegurar que los destinos sean sustentables y competitivos (Pearce, 2016). 
La gestión de los destinos es un tema de creciente importancia en la medida que los destinos compiten para obtener los niveles más elevados de calidad en la experiencia turística integral de los visitantes; y necesitan gestionar los impactos del turismo en la población local y en su medio ambiente (Moreno Melgarejo, Sariego López, \& Ávila Bercial, 2018).

Ante los nuevos desafíos impuestos por el desarrollo progresivo y permanente de las tecnologías de la información y la comunicación; así como cambios en las tendencias de la demanda turística, se necesitan nuevos enfoques en la gestión de los destinos y la concepción de destino turístico inteligente es una nueva y valiosa manera de gestión, por lo que el reto actual es transformar los destinos turísticos en destinos turísticos inteligentes.

(Romero, 2017) define un destino turístico inteligente (DTI) como un "destino innovador, consolidado sobre una infraestructura tecnológica de vanguardia, que garantiza el desarrollo sostenible del territorio turístico, que promueve la accesibilidad universal, que facilita la interacción e integración del visitante con el entorno e incrementa la calidad de su experiencia en el destino y mejora la calidad de vida de los residentes".

Los destinos inteligentes constituyen una herramienta fundamental para el desarrollo sostenible y no sólo aportan un impulso al sector turístico, sino a las sociedades en general. La apropiación y uso de las soluciones tecnológicas contribuyen de manera efectiva a que las decisiones puedan tomarse en el mismo territorio turístico sobre una base empírica, toda vez que permite priorizar diversas acciones y medidas anticipando futuros escenarios, premisa fundamental para una gestión responsable del turismo y sus consecuentes impactos (Salessi, 2017).

López, et.al (2015) definen los principios para convertir un destino turístico en DTI, los mismos son:

1. Compromiso y voluntad de cambio

2. Viabilidad financiera. Búsqueda de financiación

3. Colaboración público - privada

4. Participación del ciudadano y el turista.

Los propios autores también plantean que el principal motor de la transformación es la voluntad de cambio. "Hay que ser conscientes de la necesidad de evolucionar y tener la firme voluntad de poner en marcha una estrategia concreta, así como los recursos para llevarla a cabo, tanto desde el lado público como del conjunto de la sociedad y la empresa privada. Por ello, se debe comenzar con un informe diagnóstico que identifique las necesidades en los distintos ámbitos del municipio y cuantifique las intervenciones necesarias, así como los elementos regulatorios y de planificación que se requieren para su implementación".

Impulsar la creación de DTI requiere que la iniciativa pública lidere el proceso y se haga de forma planificada. Existe un acuerdo general sobre el hecho de que los destinos que queden al margen de este proceso pueden perder muchas opciones de competir con éxito 
con el resto pues perderán las ventajas competitivas basadas en la mejora de la imagen, que poseen los DTI (González Reverté, Díaz Luque, Gomis López, \& Morales Pérez, 2018).

Algunas de las características que un destino inteligente podría cumplir, enfocadas tanto en la sociedad como en las empresas y el gobierno son: vialidades para ciclistas, banquetas para transeúntes, manejo de aguas residuales, manejo de la basura y reciclado, uso de redes sociales y página web, internet abierto en las principales vialidades y sitios turísticos, además de placas con código QR, uso de aplicaciones y realidad aumentada (Piñón-González \& Castillejos-López, 2019).

\section{Metodología}

Diversos autores han abordado los indicadores o áreas de actuación a considerar para convertir un destino turístico en un DTI, entre ellos: (Acosta, 2017; Asociación-Españolade-Normalización, 2018; Boes, Buhalis, \& Inversini, 2015; Gajdošík, 2018; INVATTUR, 2015; Ivars-Baidal et al., 2016; Ivars Baidal, Celdrán Bernabeu, \& Femenia Serra, 2017; Khan, Woo, Nam, \& Chathoth, 2017; López et al., 2015; López de Ávila Muñoz \& García Sánchez, 2014; Romero- Rodríguez, Aragonés-Beltrán, Vivancos, \& Molina Pons, 2018; SEGITTUR, 2015) (Figura 1).

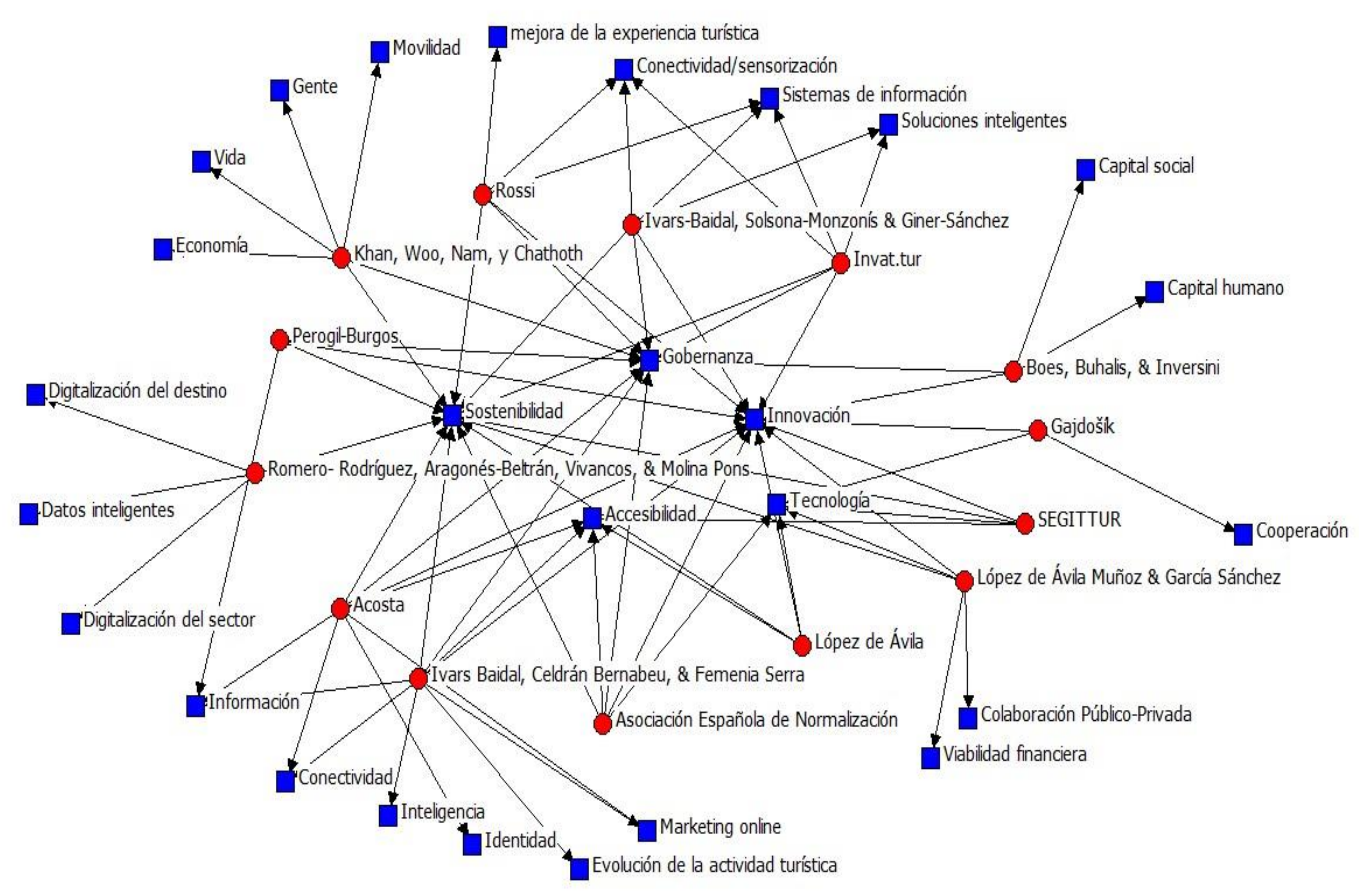

Figura 1. Red de indicadores y autores de DTI. Fuente: Elaboración propia a partir del software Ucinet for Windows

De la conceptualización realizada, a partir de las propuestas de los autores anteriores, se obtuvieron las variables que categorizan los destinos turísticos inteligentes. A partir de 
un análisis de correlaciones entre variables, tomando como escala de similitud binaria el método Jaccard, se obtuvo que las variables que mayor grado de centralidad normalizada poseen en relación a las demás son: tecnología, innovación, sostenibilidad, accesibilidad y gobernanza. De igual forma, estas variables presentan menor centralización global de cercanía con la red; mayor centralización global de la cercanía armónica y mayor robustez en los valores el eigenvector como medida de centralidad (Tabla 1).

Tabla 1. Análisis de centralidad de las variables estudiadas. Fuente: Elaboración propia a partir de salidas UCINET

\begin{tabular}{|l|r|r|r|r|r|r|r|}
\hline ID & Degree & Betweenness & Closeness & HarmonicCloseness & Eigenvector & 2-Local Eigenvector \\
\hline Sostenibilidad & 17 & 16,167 & 35 & 17 & 0,328 & 163 \\
\hline Gobernanza & 16 & 10,917 & 36 & 16,5 & 0,32 & 160 \\
\hline Innovación & 15 & 8,483 & 37 & 16 & 0,309 & 156 \\
\hline Tecnología & 15 & 8,483 & 37 & 16 & 0,309 & 156 \\
\hline Accesibilidad & 15 & 8,617 & 37 & 16 & 0,307 & 155 \\
\hline
\end{tabular}

A partir del resultado anterior se determinaron los indicadores o variables sobre las que se realizará el diagnóstico inicial del destino y luego se identificaron los items correspondientes a las mismas a partir de las propuestas de (INVATUR, 2015) e (Ivars Baidal, 2017). Las variables e ítems definidos se describen a continuación.

Gobernanza: Funcionamiento del portal del ciudadano de los gobiernos municipales, verificación de los servicios que ofrecen las empresas y organismos de la economía por Internet y disponibilidad financiera para el desarrollo del gobierno electrónico.

Innovación: Capacidad de liderazgo para gestionar la mejora de la experiencia turística, existencia y efectividad de los sistemas de información para medir los indicadores de resultados en las organizaciones turísticas y, visión compartida consensuada entre los principales actores del destino

Tecnología: Cualidades del sistema de información y promoción turística (accesibilidad a información turística pública a través de dispositivos inteligentes), gestión de plataformas sociales, existencia de plan de comunicación y marketing online, existencia de una oficina de información turística digital, sistema de reservas y plataforma tecnológica integrada de datos para la gestión del destino.

Sostenibilidad: Nivel de desempleo, productividad media, alcance de las redes de distribución de agua y saneamiento, alcance de las redes de distribución de energía y uso de energías renovables.

Accesibilidad: Accesibilidad al espacio del destino por diferentes vías de transporte y accesibilidad a las ofertas y servicios a través de plataformas inteligentes. 
Para evaluar la situación del destino Holguín en cada uno de los indicadores definidos se utilizaron diversos métodos, como: observación, revisión documental, entrevistas, criterio de especialistas que otorgaron una puntuación en una escala de Likert de cinco categorías, donde uno fue ausente y cinco: presente con adecuada concepción y efectividad. De acuerdo a (Esteban \& Fernández, 2017) el uso de esta escala tiene un grupo de ventajas, entre las cuales resaltan: fácil de diseñar y comprender, respuestas muy fáciles, no requieren conocimientos especiales y tienen muchas posibilidades de tratamiento cuantitativo.

\section{Resultados y discusión}

La aplicación de los métodos y herramientas descritas en el apartado anterior en el año 2019, en el sector turístico y también en el gobierno y otras organizaciones públicas que se involucran necesariamente en la gestión del destino turístico de Holguín, permitió identificar la situación actual del mismo en relación con los indicadores definidos, los que se describen seguidamente.

El portal del ciudadano del Gobierno de Holguín es un sitio informativo e interactivo, está conformado por los apartados: Ciudadanía (deberes y derechos, documentos legales, concursos y convocatorias), Gobierno (revista Holguín, estructura de la Asamblea Provincial del Poder Popular), Trámites (Planificación Física, Vivienda, ONAT, licencia de conducción, Inmigración) y existe un apartado de Diálogo donde se pueden escribir comentarios y consultas.

Algunas entidades ofrecen servicios on line. La ONAT posee una herramienta que calcula el impuesto sobre los ingresos personales en el sitio web www.mfp.cu; ETECSA y el Banco Central de Cuba también poseen servicios on line, sin embargo, otras entidades como: Planificación Física, Dirección de Viviendas y Empresa Eléctrica no disponen aún de servicios on line.

Es limitada la disponibilidad financiera para el desarrollo de una gobernanza electrónica, aunque el Gobierno Central ha dado una alta prioridad para esta actividad en el corto y mediano plazo, por lo cual este indicador se debe transformar favorablemente.

La capacidad de liderazgo de los actores turísticos para gestionar la mejora de la experiencia turística es limitada (tres en la escala de uno a cinco) y también la existencia y efectividad de los sistemas de información para medir los indicadores de resultados (puntuada por los especialistas con tres de promedio). Así como la visión compartida consensuada entre los principales actores del destino (evaluada con tres en la escala señalada).

El sistema de información y promoción turística, liderada por INFOTUR, tiene como cualidades la presencia en Facebook, Twitter y YouTube; así como en el portal holguintravel. Se interactúa con los clientes únicamente en Facebook y solo existe accesibilidad a información pública a través de dispositivos inteligentes dentro de Cuba 
en las zonas con conexión a Internet. No se gestiona la reputación on line en sitios de comentarios de viajeros.

El comportamiento de otros indicadores de la gestión turística se refleja en:

- Existe un plan de comunicación y marketing del destino que incluye acciones digitales, pero no es suficientemente abarcador

- No existe una Oficina de Información Turística digital. En INFOTUR se asumen algunas tareas en esta dirección

- Baja presencia de reservas on line en organizaciones turísticas estatales y no estatales y limitaciones importantes para realizarlas a través de dispositivos inteligentes desde Cuba, por insuficiente conectividad

- No existe una plataforma tecnológica integrada de datos para la gestión del destino

Por otra parte, los resultados obtenidos en relación con indicadores sociales son los siguientes:

- $\quad$ El 65,3 por ciento de la población tiene acceso a redes de distribución de agua

- Están electrificadas por diferentes fuentes el 100 por ciento de las viviendas

- Solo se desarrollan fuentes de energía renovable en Gibara, no así en el resto del territorio

El destino Holguín posee buena accesibilidad aérea, a partir de la existencia de un aeropuerto internacional que recibe vuelos desde varios continentes. Así mismo dispone de un sistema vial que lo enlaza con el resto de los destinos cubanos, aunque con insuficiente estado técnico de sus carreteras.

A partir de esta información se pudo precisar que las variables con resultados favorables para la gestión del destino Holguín como un destino turístico inteligente se refieren a la accesibilidad, la infraestructura eléctrica y el inicio y prioridad estatal del desarrollo del gobierno electrónico en los municipios.

Así mismo los indicadores que requieren transformación para que el destino se convierta en un destino turístico inteligente se resumen en:

- Insuficiente capacidad de liderazgo de los actores turísticos para gestionar la mejora de la experiencia turística, lo que expresa una brecha en la voluntad de cambio

- Limitada disponibilidad financiera para el desarrollo de una gobernanza electrónica y la ampliación de la oferta de servicios on line

- Baja conectividad a Internet, a partir que sólo se accede desde los sitios wifi

- Escaso alcance de las redes de distribución de agua.

\section{Conclusiones}

Una vez concluida la investigación se arribó a las conclusiones siguientes: 
- Existen limitaciones en el destino de Holguín en los dos principios fundamentales para convertir el mismo en un DTI: voluntad de cambio y disponibilidad financiera

- Los gestores turísticos no aprovechan suficientemente las oportunidades existentes para una mejora en la gestión de la experiencia turística

- La infraestructura tecnológica general y en particular la de información y comunicaciones; no responde a los requerimientos de un destino inteligente

- Las principales fortalezas de Holguín para convertirse en un destino turístico inteligente están relacionadas con la existencia de infraestructuras importantes para la accesibilidad y redes eléctricas; así como el inicio y prioridad estatal para el desarrollo del gobierno electrónico.

\section{Referencias bibliográficas}

Acosta, D. G. (2017). Smart City, el puntapié para el desarrollo inteligente del turismo en un destino urbano. Universidad Nacional de La Plata, Buenos Aires.

Asociación-Española-de-Normalización. (2018). UNE 178501 Sistema de Gestión de los destinos turísticos inteligentes. Requisitos. Madrid

Boes, K., Buhalis, D., \& Inversini, A. (2015). Conceptualizing smart tourism destination dimensions.

Femenia-Serra, F., \& Ivars-Baidal, J. A. (2018). Smart tourism: Implicaciones para la gestión de ciudades y destinos turísticos. Valencia, 129 - 151.

Fernández Alcantud, A. (2017). Innovación y destinos inteligentes: Oportunidad para el know how turístico español. SEGITTUR.

Gajdošík, T. (2018). Smart Tourism: Concepts and Insights from Central Europe. Czech Journal of Tourism, 25-44. doi:10.1515/cjot-2018-0002

González Reverté, F., Díaz Luque, P., Gomis López, J. M., \& Morales Pérez, S. (2018). Reflexiones sobre la percepción de los Destinos Turísticos Inteligentes españoles por parte de los actores turísticos. ARA, 8(1), 21-35.

INVATTUR. (2015). Manual Operativo para la confiuración de destinos inteligentes.

Ivars-Baidal, J. A., Solsona-Monzonís, F. J., \& Giner-Sánchez, D. (2016). Gestión turística y tecnologías de la información y la comunicación (TIC): El nuevo enfoque de los destinos inteligentes. Documents D'anàlisi Geogràfica, 62(2), 327 346.

Ivars Baidal, J. A., Celdrán Bernabeu, M. A., \& Femenia Serra, F. (2017). Guía de implantación de destinos turísticos inteligentes. 
Khan, M. S., Woo, M., Nam, K., \& Chathoth, P. K. (2017). Smart city and smart tourism: A case of Dubai. Sustainability, 9. doi:10.3390/su9122279

López, A., Lancis, E., García, S., Alcantud, A., García, B., \& Muñoz, N. (2015). Informe destinos turísticos inteligentes: construyendo el futuro.

López de Ávila Muñoz, A., \& García Sánchez, S. (2014). Destinos Turísticos Inteligentes. SEGITTUR.

Moreno Melgarejo, A., Sariego López, I., \& Ávila Bercial, R. (2018). La planificación y la gestión como herramientas de desarrollo de los destinos turísticos. Turismo y Desarrollo. doi:http://hdl.handle.net/20.500.11763/turydes25gestion-destinos

Pearce, D. G., 25(1), 1-16. (2016). Modelos de gestión de destinos. Síntesis y evaluación. Estudios y Perspectivas en Turismo, 21(1).

Piñón-González, M. A., \& Castillejos-López, B. (2019). Huatulco desde la perspectiva de los destinos inteligentes

Romero- Rodríguez, C., Aragonés-Beltrán, P., Vivancos, J.-L., \& Molina Pons, F. (2018). Requerimientos Funcionales para la Gestión de los Destinos. Paper presented at the II Congreso Mundial de la OMT sobre Destinos Turísticos Inteligentes.

Romero, C. (2017). Destinos Turísticos Inteligentes (DTI): Visión Estratégica.

Salessi, M. L. (2017). Destinos Turísticos Inteligentes: Una perspectiva desde la inclusión social y la participación comunitaria. Paper presented at the Actas del Seminario Internacional Destinos Turísticos Inteligentes: nuevos horizontes en la investigación y gestión del turismo.

SEGITTUR. (2015). Informe destinos turísticos: construyendo el futuro.

\section{Ciencia
Digital
Editual}




\section{PARA CITAR EL ARTÍCULO INDEXADO.}

Medina Labrada, J. R., Pérez Ricardo, E. del C., \& Batista Sánchez, E. (2021). Hallazgos iniciales de la evaluación de Holguín para su desarrollo como destino turístico inteligente. Explorador Digital, 5(1), 121-132. https://doi.org/10.33262/exploradordigital.v5i1.1493

\section{¿Ciencia}

El artículo que se publica es de exclusiva responsabilidad de los autores y no necesariamente reflejan el pensamiento de la Revista Explorador Digital.

El artículo queda en propiedad de la revista y, por tanto, su publicación parcial y/o total en otro medio tiene que ser autorizado por el director de la Revista Explorador Digital.
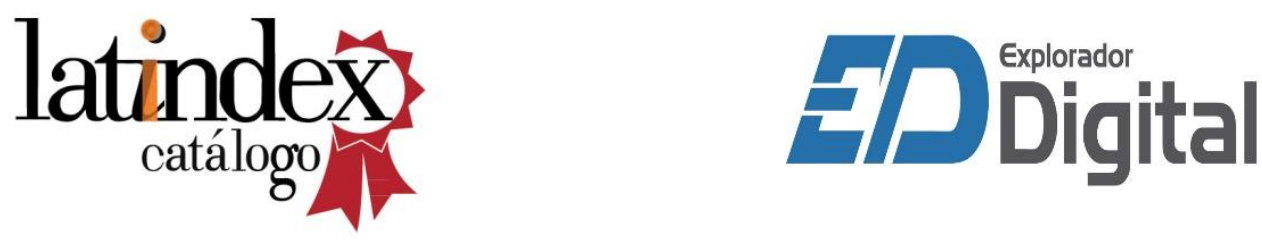